\title{
Current Elemental Speciation Analysis from a Green Chemistry Perspective
}

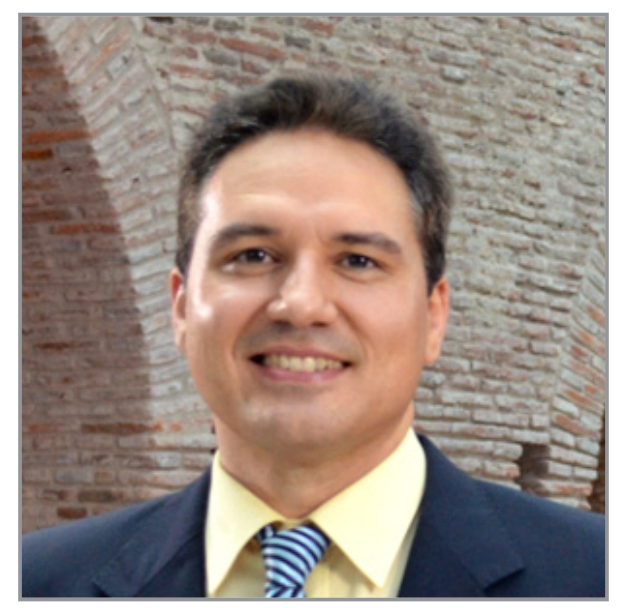

Rodolfo G. Wuilloud (1D $ه$

Associate Professor

Laboratory of Analytical Chemistry for Research and

Development (QUIANID)

Interdisciplinary Institute of Basic Sciences (ICB-CONICET

UNCUYO)

Faculty of Exact and Natural Sciences

National University of Cuyo

Mendoza, Argentina

There is no doubt that elemental speciation is a concept that is extremely relevant in nutritional, toxicological and environmental studies, as it generates crucial information enabling the full understanding of the bioavailability and toxicity of an element. However, high selectivity and sensitivity are the main demands to be covered by analytical methods for trace element speciation studies. To accomplish these goals, the combination of a selective separation technique with highly sensitive detectors has been the main strategy. Although any kind of separation technique is feasible, chromatographic techniques are easily coupled to elemental-specific detectors. Likewise, non-chromatographic separation techniques can also be used for speciation analysis, but with a more limited separation power. In any case, both methodological approaches require constant improvements due to the high complexity of analyzing the speciation of some elements and the difficulty imposed by sample matrices.

Among these general strategies, the use of the so-called hyphenated instrumental techniques has gained much terrain in the speciation arena over the years with respect to those methods involving nonchromatographic techniques for the separation of chemical species. This is mainly due to the greater practicality, automation and analytical frequency that is generally provided by the former. However, and although increasing research is currently being carried out on green analytical chemistry, there is still little critical reflection in the scientific community regarding the environmental sustainability of the practices involved in elemental speciation analysis and the need to address this concept during the development of this type of analytical method. For example, the HPLC-ICP-MS instrumental coupling has been heavily applied as a tool in speciation studies, but it can be criticized from an environmental point of view if we consider the fact that significant amounts of solvents and chemical reagents are used to separate the species of a particular element or a group of elements. Likewise, the low efficiency of the nebulization process required for sample introduction into ICP-MS is responsible for significant waste generation after several samples analyzed with this instrument. Also, this instrumentation has a high demand of energy for its operation, a relatively high cost and the need for highly trained personnel, issues that sometimes pose great challenges in Latin American countries due to the usual economic difficulties. At this point, a first reflection arises about the need to involve the concept of green chemistry during the design of analytical instrumentation, so that the equipment developed promotes the care of the environment and not only prioritizes the productivity of laboratories. In this sense, without a doubt, the companies that manufacture analytical instrumentation have a great responsibility and will only 
change their priorities if there is a regulatory framework derived from the policies imposed by each country and its commitment to preserve our environment.

In contrast, it can be considered that elemental speciation analysis based on the application of non-chromatographic separation techniques has had greater development in the sense of including the concept of green or sustainable chemistry. In this way, the emergence of liquid or solid phase microextraction techniques, where the use of solvents is avoided or at least minimized significantly, implies great environmental benefits, in addition to the possibility of separating chemical species and even increasing analytical sensitivity through the pre-concentration advantage. In addition, reducing the scale of analytical operations has allowed small volumes of solvents resulting from techniques such as LLME and SPME to be completely injected into elemental detectors such as ETAAS, which can also decompose the injected solvents during the pyrolysis stage. This vast universe of microextraction techniques has also addressed the concept of green chemistry through innovations regarding the use of more ecological solvents, more efficient sorbent materials that pose a lower need for their use and the possibility of their recycling.

Obviously, non-chromatographic techniques have a limited separation capacity when compared to HPLC or others. With these few words, I do not intend to position one above the other, but they can be considered a very attractive option for laboratories that have a high requirement for analysis and the need to determine few species, since they avoid the generation of significant amounts of waste, demand a smaller volume of solvents and may require less expensive analytical instrumentation. The choice of a specific technique from a group of elemental speciation analysis techniques will depend on several factors, among which the complexity of the speciation and the required analytical sensitivity will play a crucial role. However, when choosing, I also appeal to analysts to consider how much environmental sustainability is implicit in our daily practices, if not for us, at least for future generations. 